\title{
Ocupaciones humanas del Holoceno tardío y tecnologías de caza en la localidad arqueológica Laguna Azul (meseta de Somuncurá, Río Negro, Argentina) \\ Late Holocene human occupations and hunting technologies at the Laguna Azul site (Somuncurá plateau, Río Negro, Argentina)
}

\author{
Virginia Lynch' (1) | Darío Omar Hermo (1) | Laura Lucía Miottil (1) \\ Universidad Nacional de La Plata. La Plata, Buenos Aires, Argentina
}

\begin{abstract}
Resumen: En este trabajo se realiza una primera caracterización de los diseños de puntas de proyectil registradas en el sitio Laguna Azul, Parapeto 3 (LA-P3), localizado en el macizo de Somuncurá (Río Negro, Argentina). El objetivo es dar a conocer las tecnologías de caza implementadas por los grupos cazadores-recolectores que habitaron el sitio durante el Holoceno tardío (desde ca. 1900 años AP), a partir del análisis de diferentes variables del diseño, teniendo en consideración la aerodinámica, superficie de refuerzo y tipo de enmangue de acuerdo con el modelo de asignación funcional propuesto por Ratto (2003). Sumado a esto se realizaron análisis complementarios de microdesgaste que permitieron incrementar la información con relación a las formas y materiales empleados en el sistema enastilante. A partir de estos análisis, se identificaron micropulidos y residuos en sectores basales y limbo-proximales que indicarían indicarían el proceso de armado de cabezales líticos analizados; mientras que los estudios del diseño permitieron reconocer por un lado, diferentes estadios de manufactura (preformas, formas básicas, mantenidas y recicladas), y por el otro, el uso de por lo menos dos sistemas técnicos para la captura de presas: arco y flecha y lanza arrojadiza a mano y/o dardo.
\end{abstract}

Palabras-clave: Tecnología de caza. Norpatagonia. Holoceno tardío. Análisis de microdesgaste.

Abstract: Here we present an initial characterization of projectile point designs recovered from the Laguna Azul site, Parapet 3 (LA-P3), located in the Somuncurá Massif (Río Negro, Argentina). Our goal was to investigate the hunting technologies implemented by the hunter-gatherer groups that inhabited this site during the Late Holocene (ca. 1900 years BP). We analyzed different design variables and considered aerodynamics, reinforcement surface, and shaft system according to the functional model proposed by Ratto (2003). Supplementary micro-wear analyses were also conducted in order to generate additional data related to the shapes and materials utilized in the shaft system. These analyses identified black micro-residues in the basal sectors, while macroscopic assessments of the design manufacture indicated that different manufacturing stages were involved (pre-shaping, basic forms, maintenance, and recycling) as well as the use of at least two technical systems used to capture prey (bow and arrow and throwing spears or darts).

Keywords: Hunting technology. North Patagonia. Late Holocene. Micro-wear analysis.

Lynch, V., Hermo, D. O., \& Miotti, L. L. (2020). Ocupaciones humanas del Holoceno tardío y tecnologías de caza en la localidad arqueológica Laguna Azul (meseta de Somuncurá, Río Negro, Argentina). Boletim do Museu Paraense Emílio Goeldi. Ciências Humanas, 15(1), e20190088. doi: 10.1590/2178-2547-BGOELDI-2019-0088

Autor de correspondencia: Virginia Lynch. Universidad Nacional de La Plata. Facultad de Ciencias Naturales y Museo. Paseo del Bosque, s./n. La Plata, Buenos Aires, Argentina. 1900 (lynchvirginia@gmail.com).

Recibido el 30/08/2019

Aprobado el 20/12/2019 


\section{INTRODUCCIÓN}

La cacería es una actividad importante en la reproducción social y consiste en la obtención de recursos faunísticos silvestres por parte de las poblaciones humanas basada en una diversidad de aspectos (subsistencia, económicos, políticos, sociales etc. - ver (Sidéra et al., 2006). Los encuentros entre los cazadores y las presas se dan en un ámbito significativo, donde esas relaciones se viven cotidianamente. Este habitar, donde se materializan los encuentros, genera un paisaje o locus de caza, cuyas huellas pueden identificarse a partir de metodologías propias de la arqueología, para generar distintas interpretaciones en relación a las prácticas cinegéticas a lo largo de la historia. Es así que, las estructuras (refugios y/o escondites), conforman junto con determinados rasgos topográficos del entorno, un paisaje que permite el encuentro entre cazador y presa, a partir de las relaciones y los conocimientos adquiridos en la misma práctica (Odell \& Cowan, 1986; Ingold, 1993; Binford, 1981; Churchill, 1993; Aschero et al., 1991; Aschero \& Martínez, 2001; Ratto, 2003, 2013).

Dicha actividad consiste en la planificación, conocimientos previos y desarrollo de estrategias, sumado a tareas productivas como la elaboración y uso de diferentes tecnologías. El instrumental adecuado para la caza, implica estrategias tecnológicas amplias que involucran el aprovisionamiento de materias primas de distinto origen (rocas, madera, plumas, tendones), técnicas de trabajo particulares y la sistematización de actividades de producción. Estos sistemas de captura comprenden, por lo tanto, una manufactura anticipada de los elementos que los componen (Aschero, 1988; Martínez, 2003; Ratto, 2003; Hocsman, 2007; Álvarez, 2011; Rosero Moreno, 2011).

El sitio bajo estudio forma parte de la localidad arqueológica Laguna Azul (LA) en el noreste del macizo de Somuncurá. Sobre las bardas basálticas que bordean al norte de la laguna se identificaron diferentes estructuras de piedra, muchas de ellas definidas como parapetos (Miotti et al., 2014). Debido a la cantidad de cabezales líticos identificados en el Parapeto 3 del sector 1 (LA-P3) el siguiente trabajo se encuentra focalizado en esta estructura de bloques basálticos de morfología semicircular. El sitio ha sido definido como de actividades múltiples con evidencia de equipamiento para sucesivas reocupaciones y entre estas actividades, se ha podido inferir la manufactura y mantenimiento de puntas de proyectil. Hasta el momento, los sistemas de armas relacionados con LA-P3 han sido definidos como bolas de boleadora y sistemas con puntas (Miotti et al., 2014, 2016; Lynch \& Terranova, 2019). En este trabajo nos focalizamos en la variabilidad técnicomorfológica y morfológico-funcional de los cabezales líticos registrados, lo que permitirá incrementar la información respecto a las tecnologías de caza utilizadas por las sociedades que habitaron Somuncurá durante el Holoceno tardío (desde ca. 1900 años AP). Asimismo, se propone identificar la variabilidad presente en el conjunto, teniendo en consideración las secuencias de manufactura, posibles usos, mantenimiento, descarte y los procesos post-depositacionales que pudieron afectarlas.

\section{ÁREA DE ESTUDIO Y PRINCIPALES CARACTERÍSTICAS DEL SITIO}

La meseta de Somuncurá se halla entre los $66^{\circ}$ y $68^{\circ}$ de longitud $O$ y $41^{\circ}$ y $43^{\circ}$ de latitud $S$, y abarca las provincias de Chubut y Río Negro. Este macizo se encuentra cubierto por un manto de lava originado durante el Terciario y su paisaje corresponde a una altiplanicie basáltica, disectada por cañadones y cuencas endorreicas de carácter permanente como Vacalauquén y De Las Vacas o temporarias como El Ganso, La Maciega y Laguna Azul (Remesal et al., 2001).

En los trabajos de campo en Somuncurá, hemos detectado estructuras de piedra construidas con bloques de rocas basálticas. Estas estructuras constituyen la modificación antrópica más sobresaliente del paisaje y objeto de interés de cronistas, viajeros y arqueólogos, desde mediados del siglo XIX (Moreno, n.d. [s.f.]; Gradin, 1971; Claraz et al., 1988). Si bien presentan diferentes morfologías (rectas, semicirculares, anulares y monticulares, aislada o 
agrupadas y/o interconectadas) (Miotti et al., 2014, 2016), en Laguna Azul se trata principalmente de estructuras de planta semicircular y circular, vinculadas con actividades específicas como las de reparo y control, lugares de acecho y caza, o para el descarte de osamentas, así como espacios en donde se desarrollarían actividades múltiples (Gradin, 1971; Miotti et al., 2014, 2016; Lynch \& Terranova, 2019; Vargas Gariglio et al., 2019).

El Parapeto 3 de Laguna Azul (LA-P3) forma parte de una serie de tres estructuras interconectadas con una morfología semicircular, construido a partir de una pirca seca, sin materia cementante, con una abertura hacia el Este y cuyas dimensiones no superan los ca. $3 \mathrm{~m}$ de cuerda y $2 \mathrm{~m}$ de profundidad (Figura 1). Hacia el centro de esta estructura se planteó una cuadrícula de $1 \mathrm{~m} \times$ $1 \mathrm{~m}$, de donde se identificó una secuencia estratigráfica homogénea con una potencia sedimentaria total de $50 \mathrm{~cm}$ de profundidad.

Durante la excavación de esta estructura se recuperaron abundantes materiales: líticos (principalmente raspadores y puntas de proyectil), restos faunísticos (en su mayoría de Lama guanicoe, con puntos de impacto y marcas de corte), placas grabadas, artefactos de molienda (morteros y manos de moler) y cerámica. En este último caso, se trata en su mayoría de tiestos de uso doméstico que en ciertos fragmentos registran incisiones. Hasta el momento, esta cerámica fue definida como la más temprana en este sector de meseta, lo que sugeriría, sumado a otras materialidades (placas grabadas, morteros y manos de moler), un aprovisionamiento del lugar (Miotti et al., 2014, 2016; Lynch \& Terranova, 2017, 2019; Lynch et al., 2019; Vargas Gariglio et al., 2019).

Se han obtenido dos fechados radiocarbónicos que ubican las ocupaciones del sitio durante el Holoceno tardío $[1906 \pm 26 \mathrm{BP}$ (D-007085; coal; $\delta 13 \mathrm{C}=$ $-19.8 \%$ \%) y $1756 \pm 28$ BP (D-007084; coal; $\delta 13 \mathrm{C}=$ $-20.2 \%$ )] (Miotti et al., 2014). Los niveles más profundos se encuentran en asociación con el fechado de 1906 años AP, donde se registró la mayoría de los cabezales cuyas morfologías fueron definidas como apedunculadas lanceoladas; mientras que en los niveles superiores, asociados al fechado más tardío, cabezales principalmente triangulares con pedúnculo y aletas; sin embargo un ejemplar de esta morfología fue recuperado de los niveles más profundos lo que permitiría plantear cierto sincronismo de los diseños identificados.

\section{METODOLOGÍA APLICADA}

El conjunto $(n=43)$ fue analizado mediante variables macroscópicas con el objetivo de identificar las técnicas de talla empleadas en la manufactura (Aschero, 1975, 1983), así como materias primas utilizadas y establecer su asignación funcional a los diferentes sistemas de armas (Ratto, 2003, 2013; Shott, 1997).

El análisis morfológico se efectuó teniendo en cuenta diversas variables sensu Aschero (1975, 1983): estado del conjunto, clases de rocas utilizadas, características morfológicas del limbo, pedúnculo y aletas y grupos/ subgrupos tipológicos. También se consideraron los tamaños absolutos (longitud, espesor y anchos máximos de cada pieza); al igual que ancho y espesor máximo de los pedúnculos.

El análisis de las etapas de manufactura fue realizado teniendo en consideración los estadios propuestos por Nami (1993-1994) para puntas cola de pescado y las definiciones de Aschero (1988) y Martínez (2003, p. 52) de

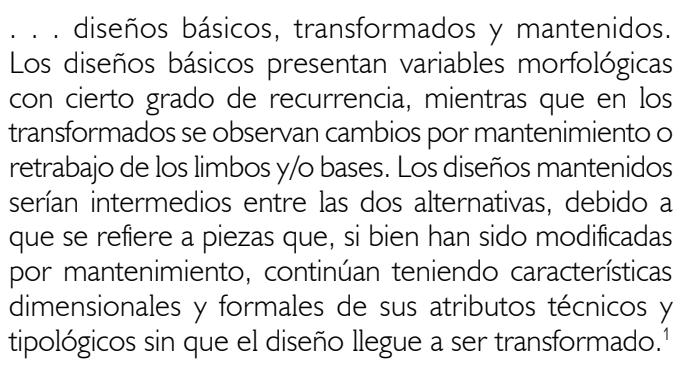

. . . diseños básicos, transformados y mantenidos. Los diseños básicos presentan variables morfológicas con cierto grado de recurrencia, mientras que en los transformados se observan cambios por mantenimiento o retrabajo de los limbos y/o bases. Los diseños mantenidos serían intermedios entre las dos alternativas, debido a que se refiere a piezas que, si bien han sido modificadas tipológicos sin que el diseño llegue a ser transformado. ${ }^{1}$

\footnotetext{
1 Ver también Hocsman (2007, 2010, 2011).
} 


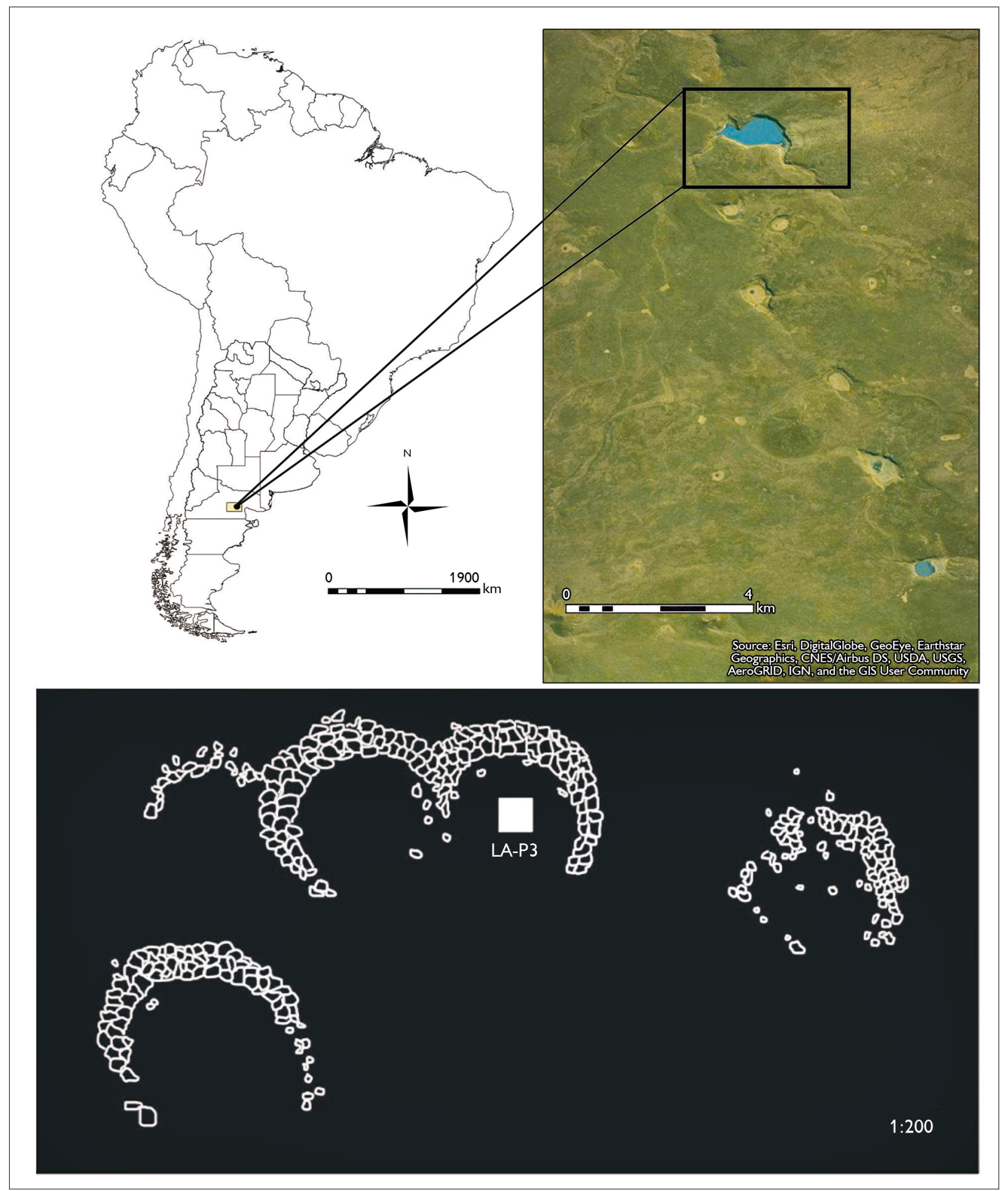

Figura 1. Mapa del área, localización del sitio Laguna Azul - Parapeto 3 (LA-P3) y detalles de las estructuras de piedra identificadas en la localidad. Fuente: Lynch (2019).

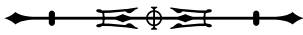


Con el objetivo de identificar los sistemas técnicos enastilantes y asignar los tipos morfológicos identificados en LA-P3 a diferentes sistemas de armas, se aplicó el modelo de asignación funcional de cabezales líticos (Ratto, 2003, 2013). En el mismo, se propone una serie de variables relacionadas a la performance del sistema técnico, tales como superficie de refuerzo², aerodinámica ${ }^{3}$, poder de penetración ${ }^{4}$ y características del enmangue ${ }^{5}$ (Ratto, 2003). Acordando con esta autora que los ". . . pedúnculos de ancho mayor a $10 \mathrm{~mm}$ podrían indicar el uso de armas de mano o arrojadizas, lanzas o dardos, mientras que los menores a $10 \mathrm{~mm}$ señalarían el empleo de armas con almacenamiento de energía (arcos y flechas)" (Ratto, 2003, p. 88, 2013, p. 90).

Por otro lado, se llevaron a cabo análisis de macro y microrrastros, para ampliar los conocimientos respecto a su posible uso y armado, mediante el registro de tipos de fracturas, puntos de impacto, estrías, características particulares del enmangue utilizado a partir del registro de sustancias adhesivas (i.e. mástic), su distribución en las piezas y micropulidos. Esto se realizó mediante un microscopio metalográfico Nikon Epiphot 200 (50x-500x) y una lupa binocular Nikon SMZ800 (10x-63x). El procesamiento y captura de imágenes fue efectuado mediante un sistema de video-microscopía Nikon Micrometrics 614.

Asimismo, se aplicó el test $U$ de Mann-Withney ${ }^{6}$ mediante el software Statistica 7.0 para determinar diferencias en las variables métricas utilizadas teniendo en consideración los diferentes diseños identificados.

\section{DESCRIPCIÓN GENERAL DE LA MUESTRA Y ANÁLISIS DEL DISEÑO DE PUNTAS}

El conjunto de LA-P3 está constituido por un total de 43 puntas de proyectil que incluye elementos enteros y fracturados. Del total, un 11\% $(n=5)$ se encuentran enteras y se registra un alto porcentaje $(66 \%, n=38)$ de elementos fracturados algunos de los cuales registran fracturas mínimas que permiten proyectar o reconstruir su forma original. Mientras que las piezas con bajo grado de integridad (bases/pedúnculos y/o ápices), se consideraron como patrón para las características diagnósticas del diseño de los ejemplares que sí pudieron ser atribuidos a algún sistema de arma.

En cuanto a la localización de la fractura, en su mayoría se ubican en diferentes sectores del limbo, aunque se identificaron mayormente en el limbo distal (37\%, n = 14) y con una disposición transversal al eje de la pieza, de morfología plana (36\%, $n=13$ ) o en charnela transversal u oblicua $(22 \%, n=8)$. También se identificaron fracturas ocasionadas por la exposición de la pieza al fuego en varios ejemplares (14\%, $n=5)$.

Las materias primas utilizadas, corresponden en su mayoría a rocas de grano fino como calcedonia, obsidiana y sílices de diferentes coloraciones (Tabla 1). Estas variedades de rocas, con excepción de la obsidiana, se han reconocido en una fuente primaria ubicada a una distancia mayor a 50 km en línea recta, en donde se identificó una cantera de grandes dimensiones, con evidencia de diferentes actividades de talla y producción de instrumental lítico. Por otro lado, se ha registrado la presencia de sílice en forma de filones en los cañadones Martel y El Rincón y fuentes secundarias en cauces temporarios y en el Bajo de El Caín (Terranova, 2013; Hermo \& Terranova, 2016). Asimismo, en la última campaña realizada en LA, fue identificada una fuente primaria de calcedonia que se denominó cantera Pazos y no se descarta la existencia de fuentes de sílices y obsidiana cercanas al sitio aún no detectadas (Vargas Gariglio et al., 2019).

2 Es aquella que soporta la fuerza de impacto o choque (Ratto, 2003).

3 En función si rige o no dicha trayectoria (Ratto, 2003).

${ }^{4}$ Se calcula en base a las medidas de los ángulos en vista plana complementada con el cálculo de área de la sección del ápice.

5 Medida del ancho de la base o del pedúnculo y teniendo en cuenta como límite $10 \mathrm{~mm}$ para distinguir entre tecnología con almacenamiento de energía o sin almacenamiento de energía.

6 Prueba no paramétrica de diferencia de medianas (Barceló, 2007).

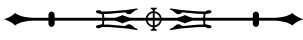


Tabla 1. Materias primas identificadas en el conjunto de cabezales líticos recuperados de LA-P3. *Se incluye la triangular apedunculada.

\begin{tabular}{c|c|c|c|cc}
\hline Grupo Tipológico & Apedunculadas & Pedunculadas & Fractura indeterminada & Total & $\%$ \\
\hline Calcedonia & $18^{*}$ & 4 & 10 & 32 & 74,4 \\
\hline Ślice & 1 & - & 5 & 6 & 14 \\
\hline Obsidiana & 1 & 2 & 2 & 5 & 11,6 \\
\hline Total & 20 & 6 & 17 & 43 & 100 \\
\hline
\end{tabular}

El uso de calcedonia, roca mayormente representada en el área de estudio, es recurrente tanto en la producción de puntas de proyectil $(74 \%, \mathrm{n}=32)$, como así también en el resto del conjunto lítico de LA-P3. Este tipo de roca es de alta dureza y suele contener gran cantidad de impurezas, lo que habría llevado a numerosas fallas, fracturas o piezas 'sin terminar'. A diferencia de esta roca, el sílex y la obsidiana (exclusivamente de coloración negra) están representadas en proporciones menores. Si bien hasta el momento, no se han hecho estudios específicos acerca de la procedencia de la obsidiana, se infiere que habría sido obtenida en el sector sur del macizo, debido a que distintas fuentes han sido identificadas en esta región, tales como Cerro Castillo, Sacanana y Sierra Negra (Stern et al., 2000).

Por otro lado, el estudio del diseño y de la morfología de las piezas permitió reconocer dos grandes grupos de acuerdo al área de enmangue (pedunculadas y apedunculadas) y a la forma perimetral del limbo (lanceolada y triangular) que coexisten en la estratigrafía de LA-P3.

\section{APEDUNCULADAS LANCEOLADAS}

Este grupo está caracterizado por un total de 19 piezas que registraron en su mayoría algún tipo de fractura (84\%, $\mathrm{n}=16$ ). La calcedonia es la roca predominante para su manufactura al igual que en la totalidad del conjunto (Tabla 1).

Para la producción de estas puntas se utilizaron diferentes formas base, entre las que se destaca el uso de lascas ( $47 \%, n=9)$, bifaces $(37 \%, n=7)$ y formas no determinables $(16 \%, n=3)$. La sección transversal del limbo fue principalmente biconvexa simétrica (58\%, $n=11)$, aunque también se reconoció plano-convexa
(26\%, $n=6)$ y biconvexa asimétrica (10\%, $n=2)$. Mientras que las bases presentaron una morfología mayormente cóncava (Figura 2A-2C).

En cuanto a las características dimensionales se trata de piezas de módulo 'grande' (Aschero, 1975, 1983) cuyas medidas promedio son: longitud de 40,3 mm, ancho de $25 \mathrm{~mm}$, espesor de $5,9 \mathrm{~mm}$ y peso de 6 grs. Las puntas fracturadas muestran promedios de 32,4 mm de longitud, ancho de $24,7 \mathrm{~mm}$, espesor de $6,7 \mathrm{~mm}$ y un peso considerable de 7,4 grs. Se realizó un test estadístico para determinar diferencias significativas entre piezas enteras y fracturadas, dando como resultado que tanto en longitud $(M-W=U=20, p>0.05)$, ancho $(M-W=U=24, p>$ $0.05)$, espesor $(M-W=U=18, p>0.05)$ y peso ( $M-W=$ $U=20, p>0.05)$ no existen tales diferencias, lo que indica que pese a estar fracturadas estos elementos mantienen las proporciones constantes de acuerdo al tipo de diseño básico. Sin embargo, llama la atención que tanto el peso como el espesor de las piezas fracturadas supere el de las enteras, lo que sugiere que podría tratarse de estadios previos a su etapa final de producción. Esto es interpretado a partir del registro de diferentes etapas de manufactura, que incluye desde primeros estadios $(16 \%, n=3)$ hasta diseños básicos (52\%, $n=10)$, mantenidos (21\%, $n=4)$ y reciclados ( $5 \%$, $n=1)$ y un solo caso indeterminado (Figura 2).

\section{TRIANGULARES CON PEDÚNCULO Y ALETAS (TPA)}

Este grupo está constituido por 6 piezas, en todos los casos se identificaron fracturas (transversal al eje morfológico rectas o en charnela oblicuas) y en su mayoría, se trata de pedúnculos con algún fragmento de limbo. A diferencia del grupo 
anterior, se evidencia un diseño básico y ausencia de diseños mantenidos o reciclados. Se seleccionaron únicamente lascas de diferentes morfologías para su producción, al igual que se identificó una sección transversal de limbo principalmente biconvexo simétrico y base cóncava del pedúnculo. De acuerdo a esto se definieron dos subgrupos: uno de bordes rectos y base cóncava y otro de bordes rectos aserrados y base cóncava atenuada (Figura 2D).
En cuanto a las características dimensionales, estas piezas son significativamente menores (longitud M-W $=U=1, p<0,05$; ancho: $M-W=U=0, p<0,05$; espesor: $M-W=U=1, p<0,05$; peso: $M-W=U=0,50$, $p<0,05)$ que el grupo de apedunculadas, y muestran dimensiones cuyas medianas son: 15,19 mm de longitud, 13,17 mm de ancho y 2,55 mm de espesor; mientras que el peso fue igualmente menor de 0,7 grs (Figura 3).

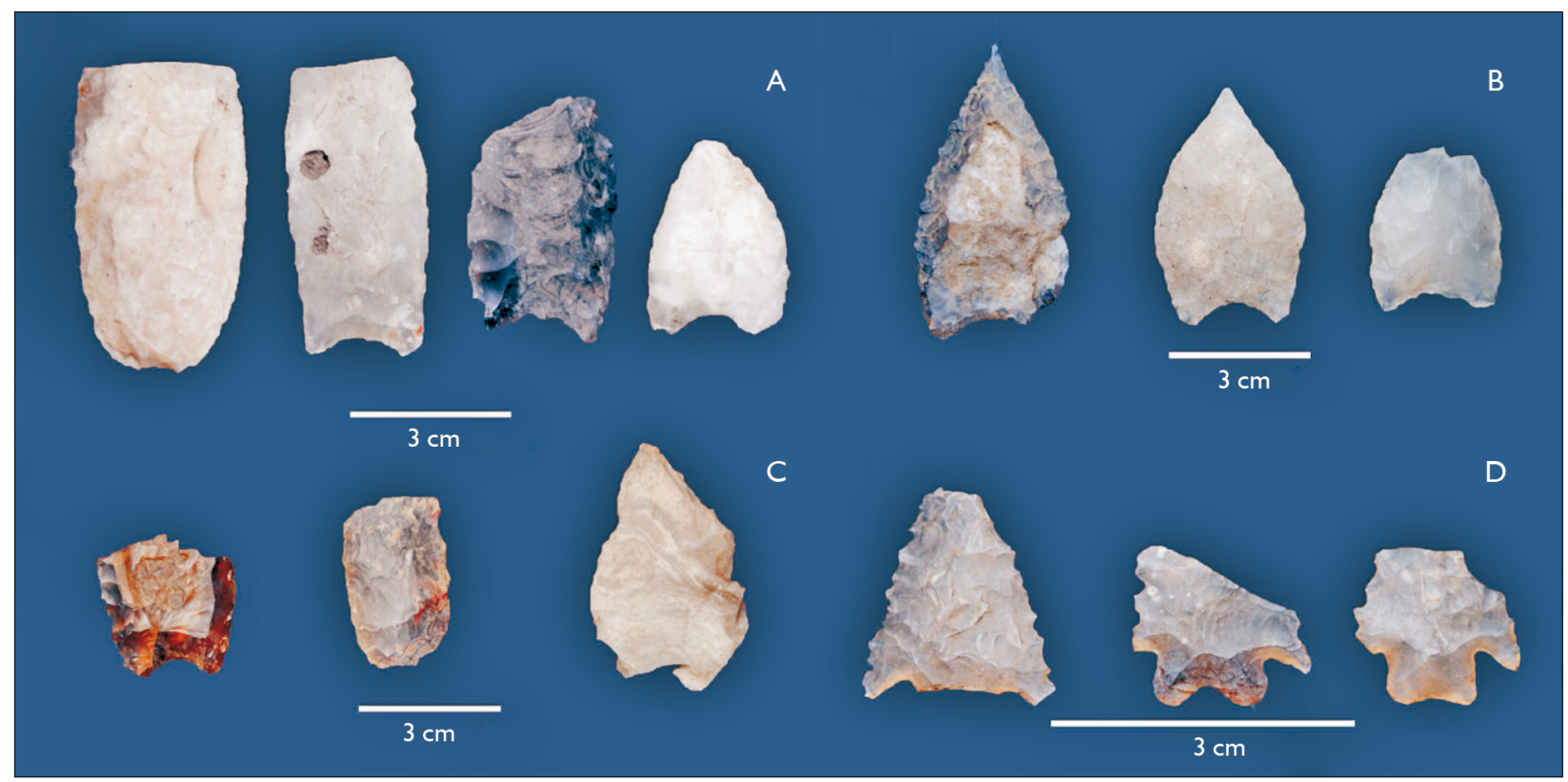

Figura 2. A-C) Diseños de puntas lanceoladas apedunculadas en diferentes estadios de manufactura. D) Diseño de puntas triangulares pedunculadas de bordes rectos aserrados. Fuente: Lynch (2019).

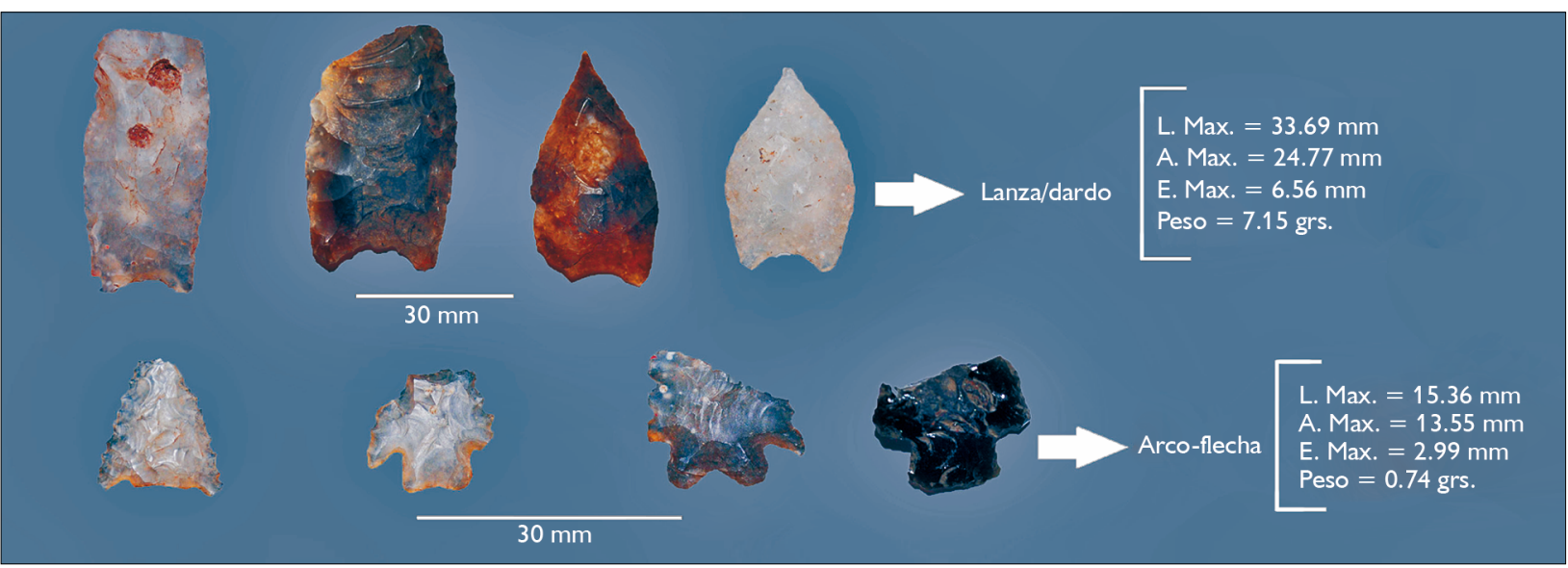

Figura 3. Diferencias métricas de los diseños identificados en LA-P3. Fuente: Lynch (2019).

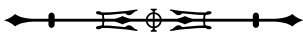




\section{APEDUNCULADA TRIANGULAR}

Se encuentra representado por un único ejemplar de bordes rectos y base cóncava. Registra una fractura en el sector del limbo distal, transversal al eje de la pieza y de morfología transversal en charnela. Al igual que el resto del conjunto, esta punta de proyectil fue elaborada en calcedonia de coloración marrón translúcido (Tabla 1). Para su manufactura ha sido utilizada una lasca como forma base y posee sección transversal biconvexa simétrica y base cóncava atenuada. Sus dimensiones son 13,17 mm de longitud, ancho de 11,26 $\mathrm{mm}$, espesor $2,36 \mathrm{~mm}$ y peso de $0,8 \mathrm{grs}$.

\section{FRAGMENTOS DE DISEÑOS \\ INDETERMINADOS}

Del conjunto analizado, 17 cabezales no pudieron ser determinados debido a que presentaron fracturas importantes en el limbo o en la raíz del pedúnculo o base. De estos elementos, nueve corresponden únicamente a ápices, tres a limbos, tres a fragmentos limbo-basales y dos a bases. Las fracturas son transversales y de sección plana $(29 \%, n=5)$, en charnela recta/oblicua $(29 \%, n=5) \mathrm{u}$ ocasionada por daño térmico (12\%, $n=2)$. Al igual que el grupo de puntas lanceoladas apedunculadas se identificó una amplia diversidad en la selección de las formas base, entre las que se hallaron bifaces, lascas y lascas laminares. Las dimensiones promedio son de 22,4 mm de longitud, $18,45 \mathrm{~mm}$ de ancho, 5,18 mm de espesor y 2,71 grs.

En cuanto a los ápices identificados $(n=9)$, presentan en su mayoría ángulos superiores a $45^{\circ}$ y con un tratamiento normal. Por otro lado, el resto de piezas indeterminadas están constituidas por fragmentos de limbo, limbo-basales y bases $(n=8)$ con sección transversal biconvexa simétrica.

\section{ANÁLISIS DEL SISTEMA DE ARMAS, EVIDENCIAS DE ARMADO Y USO POR IMPACTO}

La superficie de refuerzo es la sección del instrumento que soporta los mayores esfuerzos mecánicos, teniendo incidencia con la mayor o menor probabilidad de su fractura (es medida a partir del espesor máximo del limbo y su ancho en ese mismo sector, con valores que varían entre 0 y 1) (Ratto, 2003; Álvarez, 2011).

En los diseños tanto de puntas lanceoladas apedunculadas como de triangulares con pedúnculo se observaron valores bajos de superficie de refuerzo (84\% y $60 \%$ de los casos en cada grupo) a muy bajos (16\% y $40 \%$ respectivamente) y sección transversal principalmente biconvexa simétrica $(n=11)$ o plano convexa $(n=6)$ (Figura 4).

Por otro lado, se consideró la tenacidad de las rocas empleadas observándose un uso similar de materias primas en los diseños identificados; sin embargo, en base a estudios experimentales se sabe que la calcedonia, roca mayormente utilizada, presenta cierto grado de resistencia a las fracturas lo que le otorga una tenacidad intermedia a alta (Ratto, 2003; Lynch et al., 2019).

Respecto a la aerodinámica es fundamental para determinar si un sistema es arrojadizo y se define a partir de la interrelación entre los atributos de la sección transversal, porción de los bordes del limbo, largo y ancho de las puntas. Cuanto mayor es la superficie de contacto de la punta con el aire, mayor será la aerodinámica (Hughes, 1998). En el registro de LA-P3, las piezas identificadas como de morfología lanceoladas apedunculadas presentaron una gran superficie de contacto con el fluido y, por lo tanto, registran en su mayoría una aerodinámica imperfecta $(47,4 \%)$. Mientras que en el caso de las triangulares pedunculadas, se observó una superficie pequeña y aerodinámica perfecta para este tipo de diseño (Tabla 2).

A su vez, la morfología de las bases del grupo de lanceoladas apedunculadas, exhibió una forma principalmente cóncava $(n=7)$ y recta $(n=2)$, con un ancho de base promedio de 18,44 mm y espesor de 3,84 mm.

A diferencia de este grupo, las TPA registraron anchos de bases con dimensiones menores de 13,56 $\mathrm{mm}$ promedio y una terminación principalmente cóncava atenuada a recta (Tabla 3). 


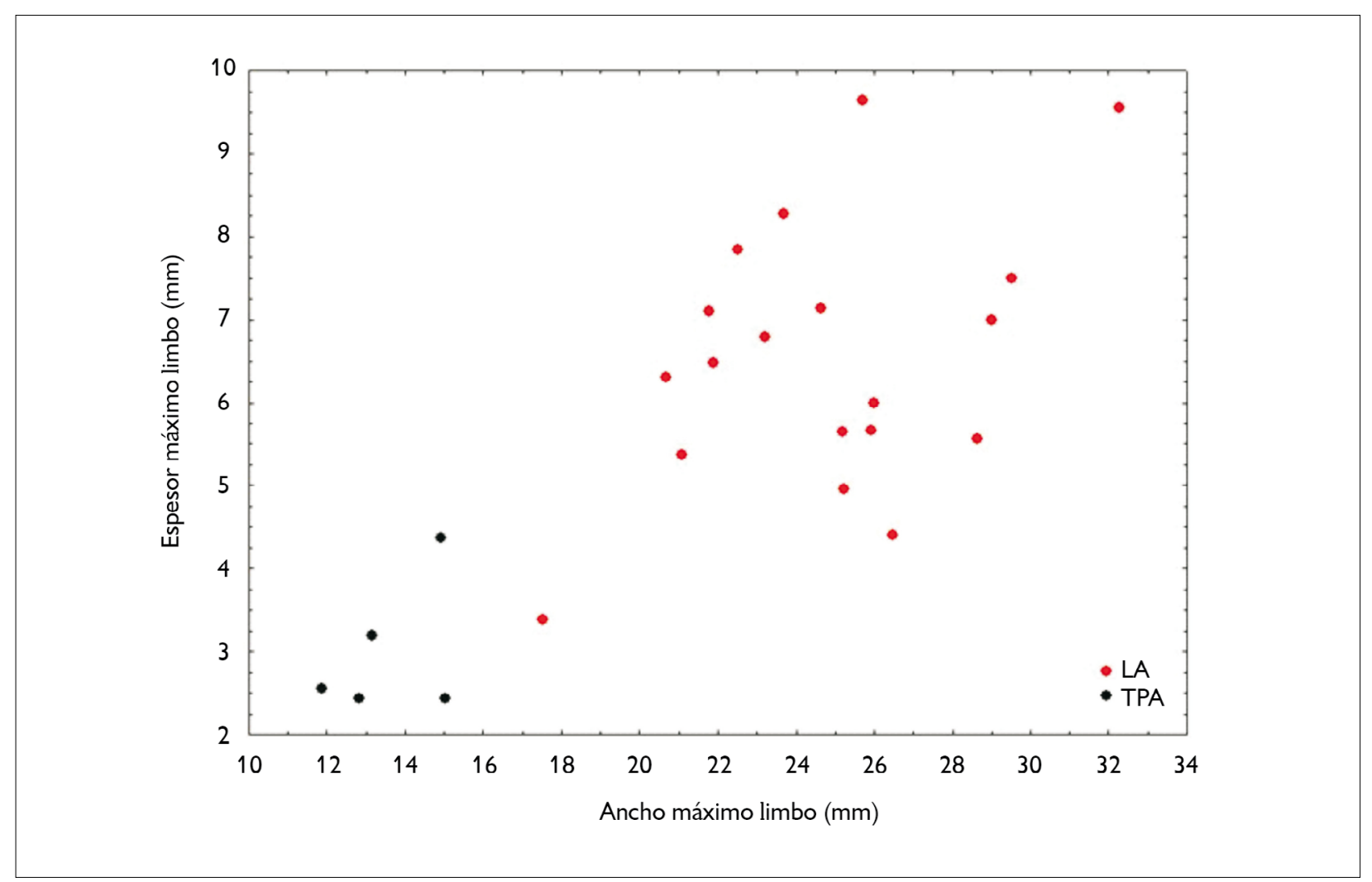

Figura 4. Relación entre el espesor máximo del limbo y ancho en ese sector para cabezales pedunculados y apedunculados. Fuente: Lynch (2019).

Tabla 2. Aerodinámica de acuerdo a los tipos de diseños identificados.

\begin{tabular}{c|c|c|c|c}
\hline \multirow{2}{*}{ Aerodinámica LA } & \multicolumn{2}{|c|}{ Lanceolada apedunculada } & \multicolumn{2}{c}{ Triangulares pedunculadas } \\
\cline { 2 - 5 } & $\mathrm{n}$ & $\%$ & 5 & $\%$ \\
\hline Perfecta & 7 & 36,8 & - & 100 \\
\hline Normal & 3 & 15,8 & - & - \\
\hline Imperfecta & 9 & 47,4 & 5 & 100 \\
\hline Total & 19 & 100 & & - \\
\hline
\end{tabular}

Tabla 3. Medidas de tendencia central de las bases en milímetros ( $\mathrm{mm})$.

\begin{tabular}{c|c|c}
\hline Ancho de bases & Apedunculadas & Pedunculadas \\
\hline $\mathrm{N}$ & 20 & 13,56 \\
\hline Media & 24,11 & 13,17 \\
\hline Mediana & 25,19 & 11,86 \\
\hline Mínimo & 11,26 & 15,01 \\
\hline Máximo & 32,28 & 1,37 \\
\hline Desv. Stand & 4,59 & \\
\hline
\end{tabular}


Por otro lado, el ángulo del ápice en vista plana indica la capacidad de penetración de una punta en la presa. Los estudios actualísticos revelan que las puntas de flechas registran ángulos inferiores a $45^{\circ}$; mientras que las que superan los $63^{\circ}$ resultan ineficientes en los diferentes sistemas técnicos (Ratto, 2003; Odell \& Cowan, 1986; Lynch et al., 2019). En el conjunto de LA-P3, el grupo de puntas de morfología lanceoladas apedunculadas presentan bases superiores a $10 \mathrm{~mm}$ y un ángulo de vista plana del ápice superior a $66^{\circ}$ promedio, concentrados entre los $55^{\circ}$ a $67^{\circ}$, lo que las ubica al límite de su vida útil. En el caso de las triangulares con pedúnculo, el ancho de las bases es menor a $10 \mathrm{~mm}$, sin embargo registran un ángulo de penetración promedio de $54^{\circ}$, lo que indica que a diferencia de las lanceoladas aún continuarían siendo útiles (sensu Odell \& Cowan, 1986).

En este sentido y a partir del análisis del diseño de las puntas recuperadas de LA-P3, podemos mencionar que el grupo de apedunculadas corresponderían a piezas con superficies de contacto relativamente 'grande', bajo a muy bajo módulo de refuerzo, diseños no aerodinámicos con bases mayores a $10 \mathrm{~mm}$ y ángulos de vista plana del ápice entre $55^{\circ}$ a $67^{\circ}$, por lo que se trataría de un diseño de dardo y/o lanza, sin almacenamiento de energía. Mientras que el grupo de TPA, caracterizado por piezas con tamaños 'pequeños' a 'medianos' y superficies de contacto variables, con un bajo a muy bajo índice de refuerzo, diseño aerodinámico con pedúnculos inferiores a 10 mm y un ángulo en vista plana promedio de $54^{\circ}$, sugiere una tecnología de puntas de flecha, es decir, con almacenamiento de energía.

En cuanto al análisis de microdesgaste de estas piezas, la observación de los bordes de limbo, ápices y pedúnculos permitió identificar en primer lugar una buena integridad de los materiales analizados, debido a que el grado de alteración de estas piezas fue leve a moderado y únicamente en cuatro ejemplares se reconocieron alteraciones severas de sus superficies, debido a la exposición directa al fuego (i.e. craquelé, fracturas internas, lustre intenso).
El análisis microscópico realizado al conjunto de puntas apedunculadas permitió detectar micropulidos generados por el contacto con material duro vegetal (madera) y residuos de coloración negra (mástic) en sectores limbo basales $(n=12)$. No se reconoció micropulido de material blando animal (i.e. cuero o piel), lo que podría deberse al escaso tiempo y forma de contacto con el material, generando micropulidos no diagnósticos y dificultando las interpretaciones respecto al sistema enastilante empleado (Franco et al., 2009). Sin embargo, la identificación de residuos (que a futuro deberán ser analizados en mayor profundidad), micropulidos de madera y el registro de diferentes etapas de producción de estos cabezales líticos, sugeriría que estos elementos probablemente hayan sido armados, utilizados, mantenidos y descartados en el sitio (Figura 5 y 6 ).

En el caso de las TPA, aunque registraron un grado de alteración leve, no se observaron micropulidos sobre las superficies de las piezas analizadas y se reconocieron fracturas en charnela oblicuas y estrías longitudinales al eje morfológico en sectores del ápice, que darían cierto sustento a un uso potencial de estas puntas $(n=3)$.

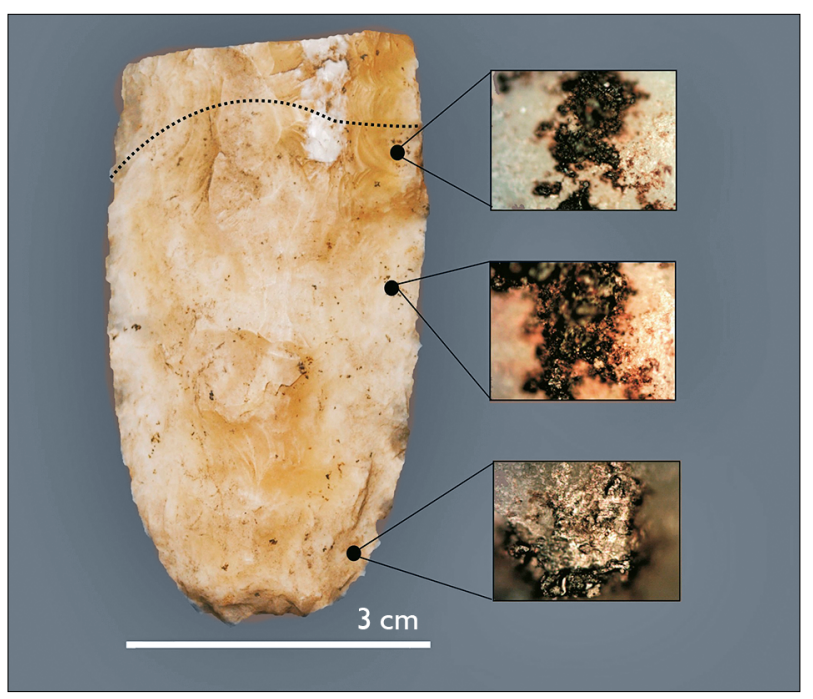

Figura 5. Punta lanceolada apedunculada con residuos de coloración negra y micropulido de material duro en sector basal y limbo proximal (magnificación 200X, campo claro). Fuente: Lynch (2019).

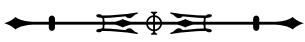




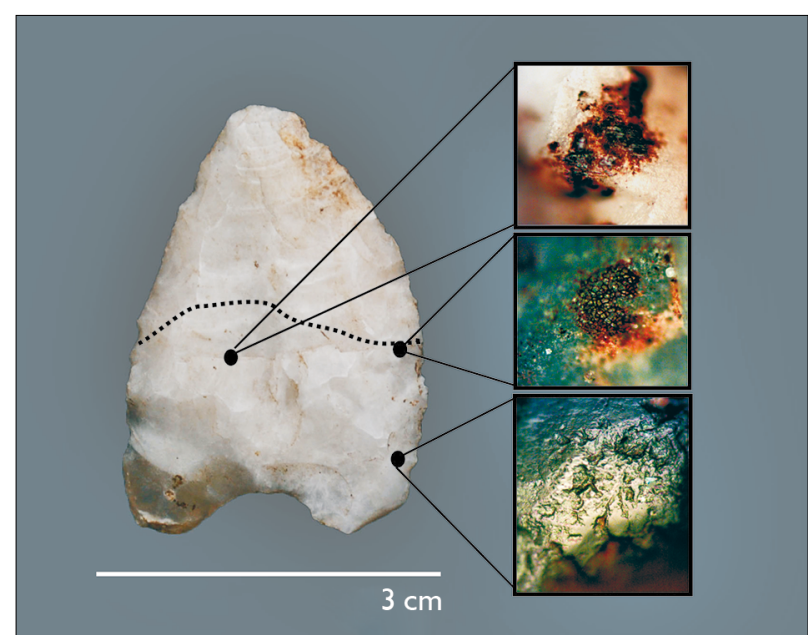

Figura 6. Punta apedunculada lanceolada reciclada (cuchillo). Identificación de residuos (mástic) y micropulido de material duro vegetal en porción basal y limbo proximal (magnificación 200X, campo claro). Fuente: Lynch (2019).

Sin embargo, estas estrías podrían haber sido igualmente ocasionadas durante el proceso de talla en la regularización del filo (estrías tecnológicas) o bien por alteraciones postdepositacionales (Keeley, 1980; Mansur-Franchomme, 1983; Álvarez, 2003, 2011).

\section{DISCUSIÓN Y CONCLUSIONES}

Laguna Azul- Parapeto 3- es una de las cinco estructuras de piedra identificadas en la localidad y ha sido definido, hasta el momento (Miotti et al., 2014), como un sitio con buena visibilidad donde se habrían desarrollado diversas actividades, tanto domésticas como vinculadas a la caza, entre ellas la manufactura y mantenimiento de puntas. Sin embargo es importante remarcar aquí, que la gran cantidad de puntas recuperadas de LA-P3 no ha sido registrada en otras estructuras excavadas en $L A$, lo que permitiría plantear un uso diferencial de las mismas. Cabe destacar a su vez, que si bien la variabilidad del conjunto lítico en LA-P3 es baja, representada principalmente por raspadores, puntas y bifaces, también fueron recuperados otros elementos tales como cerámica, placas grabadas, morteros y manos de moler desde los primeros momentos de ocupación del sitio, similar a lo que ocurre en otras áreas de Patagonia (Belardi et al., 2017; Flores Coni, 2018). A su vez, mediante el análisis arqueofaunístico se reconocieron marcas de corte en elementos asignados a Lama guanicoe y entre las partes anatómicas recuperadas se identificaron huesos de extremidades, falanges, y otros pertenecientes al cráneo (dientes y huesos del oído) (Vargas Gariglio et al., 2019).

La aplicación del modelo funcional de Ratto (2003) a los cabezales líticos de LA-P3, permitió reconocer al menos dos sistemas tecnológicos de caza: dardo y/o lanza y arco y flecha. La porción basal o pedúnculo resultó ser la parte del cabezal más representada, lo que posibilitó su asignación a sistemas de armas a través del uso de pruebas estadísticas. El descarte de estos fragmentos en LA-P3 indica que este espacio fue utilizado, entre otras actividades, para el recambio de puntas fracturadas durante cacerías, posiblemente llevadas a cabo en las inmediaciones.

En el sitio no se registraron puntas asignables a armas de mano, tecnología con poca representación en sitios del Holoceno tardío (Banegas et al., 2014). La presencia y predominio de puntas de dardo y/o lanza en el registro arqueológico de LA-P3 sugiere que habría sido la más utilizada a pesar de no ser seguro en términos de eficiencia de caza (sensu Ratto, 1994, 2003). Esta tecnología probablemente haya actuado en cacerías grupales por acecho o en desventaja por parte de las presas mediante el escondite de sus cazadores en parapetos o con camuflaje. Sin embargo, el registro sincrónico de puntas definidas de arco y flecha habría garantizado un mayor éxito y flexibilidad durante la cacería, debido a que dicho sistema es considerado seguro y que puede ser implementado tanto de modo solitario como grupal (Churchill, 1993; Ratto, 2003).

A su vez, el uso de rocas locales de buena calidad para la talla ha sido identificado para la manufactura de puntas en el sitio LA-P3. Al igual que en otras áreas (Gómez Otero et al., 2011; Prates, 2010; Ratto, 2013), las puntas de flecha fueron generadas exclusivamente a partir de lascas y mediante un mayor uso de obsidiana en su producción. 
Caso contrario, es lo observado con las puntas de dardo y/o lanza, entre las que se registró el uso de preformas bifaciales y de rocas con una mayor tenacidad, como la calcedonia, lo que brindaría un menor riesgo de fractura. Es importante mencionar que el grupo de bifaces alcanza proporciones considerables $(10 \%, \mathrm{n}=13$ del total del conjunto) y el análisis de microdesgaste efectuado a este grupo no permitió reconocer rastros de utilización efectiva sobre estos artefactos, lo que permitiría vincularlos con la producción de puntas de dardo y/o lanzas (Lynch \& Terranova, 2017, 2019).

Respecto a la disponibilidad de maderas empleadas para la confección de astiles y arcos, existe un único registro para el área. El sitio Las Cañas, localizado frente a la laguna De Las Vacas, corresponde a un enterratorio en donde se encontró un vástago de lanza de Chusquea culeou (caña coihue) con una cronología tardía de ca. 229 \pm 59 años $A P\left(\delta C^{13}\right.$-18.4 -AA85466-) (Terranova, 2013). Sin embargo, este recurso es escaso en la meseta y sería considerado como bien preciado y de acceso regional como la cordillera, lo que permitiría asumir que serían preservados y únicamente descartados en el caso de ser ineficientes. El análisis funcional realizado al resto del conjunto de artefactos líticos (en su mayoría raspadores), permitió reconocer el trabajo sobre madera (Lynch \& Terranova, 2019) lo que sugiere cierta vinculación con la manufactura de astiles, dispositivos de enmangue u otros elementos de uso cotidiano (i.e. punzones, agujas, estacas).

En síntesis, el trabajo desarrollado permitió reconocer que durante el Holoceno tardío las sociedades cazadoresrecolectores que habitaron el macizo de Somuncurá, habrían utilizado para la caza dos sistemas técnicos de armas: dardos y/o lanzas y flechas propulsadas por arco. Los antecedentes del área indican que el primer grupo habría sido incorporado en tiempos posteriores a los 2000 años AP y habría tenido una distribución amplia en el norte de Patagonia, lo que permite proponer su participación en redes de circulación e intercambios (escala regional). Mientras que el sistema de arco y flecha habría sido incorporado en momentos posteriores, desde ca. 800 años AP (Prates, 2010; Gómez Otero et al., 2011). En el caso de estudio ambos sistema coexisten con fechados de ca. 1906 a 1756 años AP, lo que plantea nuevos interrogantes a tratar con un análisis amplio de las estructuras contiguas que permita afinar las cronologías. Sin embargo, de acuerdo a estos antecedentes, el tipo de diseño identificado en las puntas de LA-P3, difiere del registro que se tiene en otros sectores de Norpatagonia. Hacia el centro y norte de Neuquén existe un predominio de puntas triangulares apedunculadas, volviéndose excepcionales las pedunculadas (Carballido Calatayud \& Fernández, 2013; Fernández \& Carballido Calatayud, 2015); esta misma situación se observa en los sitios del valle del río Colorado y sector costero de San Blas (Gómez Otero et al., 2011; Prates, 2010; Franco et al., 2009). A diferencias de esto en LA-P3 las TPA son en su mayoría de tamaño 'pequeñas' y se observa un predominio de puntas lanceoladas apedunculadas 'grandes'.

Puntas similares fueron identificadas en contextos relacionados con la industria Patagoniense, como Piedra Parada 1 (Aschero, 1983) y la capa 2 del Alero Cárdenas (Aguerre et al., 1994). "Las puntas pequeñas representarían la reducción en tamaños identificado para momentos cerámicos (posteriores a 1250 años AP) y se relacionarían con el uso de arco y flecha" (Gradin, 1980, pp. 177-194). El empleo de estos diseños desde por lo menos 2000 años AP, y que se extienden desde la costa hasta la precordillera (Prates, 2010; Bellelli et al., 2008; Gómez Otero et al., 2011; Carballido Calatayud \& Fernández, 2013; Fernández \& Carballido Calatayud, 2015) y desde el norte al sur de Patagonia, sugiere por lo tanto, la transmisión y mantenimiento de diseños exitosos.

En conclusión, la asignación funcional realizada a los cabezales líticos de LA-P3 permitió ampliar los conocimientos sobre las estrategias de caza y las decisiones tecnológicas implementadas por los grupos que habitaron la meseta de Somuncurá a lo largo del Holoceno tardío; mientras que el estudio de microdesgaste generó mayor 
información acerca de los materiales empleados en el sistema enastilante de estas puntas, al igual que de la funcionalidad del sitio.

\section{REFERENCIAS}

Aguerre, A. M., Horovitz, L., \& Patiño, V. K. (1994). Material lítico del Alero Cárdenas capa 2 y su comparación con la capa 3. En Contribución a la arqueología del Río Pinturas, provincia de Santa Cruz. Concepción del Uruguay, Argentina: Ediciones Ayllu.

Álvarez, M. R. (2003). Organización tecnológica en el Canal Beagle. El caso de Túnel I (Tierra del Fuego, Argentina) (Tesis de doctorado). Facultad de Filosofía y Letras, Universidad de Buenos Aires, Buenos Aires, Argentina.

Álvarez, M. R. (2011). Puntas de arma del extremo sur de Patagonia: Algunas consideraciones sobre diseño y contexto de uso. In J. G. Martínez \& D. L. Bozzuto (Comps.), Armas prehistóricas: múltiples enfoques para su estudio en Sudamérica (pp. 15-35). Buenos Aires: Fundación de Historia Natural Félix de Azara.

Aschero, C. A. (1975). Ensayo para una clasificación morfológica de artefactos líticos aplicada a estudios tipológicos comparativos. Informe presentado al Consejo Nacional de Investigaciones Científicas y Técnicas (CONICET). Buenos Aires.

Aschero, C. A. (1983). Ensayo para una clasificación morfológica de artefactos líticos. Apéndice A y B [Cátedra de Ergología y Tecnología]. Facultad de Filosofía y Letras, Universidad de Buenos Aires, Buenos Aires.

Aschero, C. A. (1988, octubre-noviembre). De punta a punta: producción, mantenimiento y diseño de puntas de proyectil pre-cerámicas de la Puna Argentina. In Precirculados de las Ponencias Científicas presentadas a los Simposios del IX Congreso Nacional de Arqueología Argentina, Universidad de Buenos Aires, Buenos Aires.

Aschero, C. A., Elkin, D., \& Pintar, E. L. (1991, octubre). Aprovechamiento de recursos faunísticos y producción lítica en el Pre-cerámico Tardío. Un caso de estudio: Quebrada Seca 3 (Puna Meridional Argentina). In Actas del XI Congreso Nacional de Arqueología Chilena [Tomo 2], Santiago de Chile.

Aschero, C. A., \& Martínez, J. G. (2001). Técnicas de caza en Antofagasta de la Sierra, Puna Meridional Argentina. Relaciones de la Sociedad Argentina de Antropología, 24, 215-241.

Banegas, A., Gómez Otero, J., Goye, S., \& Ratto, N. (2014). Cabezales líticos del Holoceno tardío en Patagonia meridional: diseños y asignación funcional. Magallania, 42(2), 155-174. doi: 10.4067/S0718-22442014000200009

Barceló, J. A. (2007). Arqueología y estadística (1). Introducción al estudio de la variabilidad de las evidencias arqueológicas (Collecció Materials, 187). Barcelona: Universitat Autònoma de Barcelona.
Belardi, J. B., Carballo Marina, F., Madrid, P., Barrientos, G., \& Campan, P. (2017). Late Holocene guanaco hunting grounds in southern Patagonia: blinds, tactics and differential landscape use. Antiquity, 91(357), 718-731. doi: 10.15184/ aqy. 2017.20

Bellelli, C., Scheinsohn, V., \& Podestá, M. M. (2008). Arqueología de pasos cordilleranos: un caso de análisis en la Comarca Andina del Paralelo $42^{\circ}$ y áreas vecinas durante el Holoceno Tardío. Boletín del Museo Chileno de Arte Precolombino, 13(2), 37-55

Binford, L. R. (1981). Bones: ancient men and modern myths. Nueva York: Academic Press.

Carballido Calatayud, M., \& Fernández, P. M. (2013). La caza de ungulados en el bosque de Patagonia: aportes desde la localidad de Cholila (Chubut, Argentina). Relaciones de la Sociedad Argentina de Antropología, 38(1), 59-82.

Churchill, S. E. (1993). Weapon technology, prey size selection, and hunting methods in modern hunter-gatherers: implications for hunting in the Palaeolithic and Mesolithic. Archeological Papers of the American Anthropological Association, 4(1), 11-24. doi: 10.1525/ap3a.1993.4.1.11

Claraz, J., Casamiquela, R. M., \& Hux, M. (1988). Diario de viaje de exploración al Chubut, 1865-1866. Buenos Aires: Ediciones Marymar.

Fernández, P. M., \& Carballido Calatayud, M. (2015). Armas y presas: técnicas de caza en el interior del bosque patagónico. Relaciones de la Sociedad Argentina de Antropología 40(1), 279-301.

Flores Coni, J. (2018). Poblamiento humano y uso del espacio en la meseta del Strobel (provincia de Santa Cruz): un análisis sobre la variabilidad tecnológica durante el Holoceno (Tesis de doctorado). Facultad de Filosofía y Letras, Universidad de Buenos Aires, Buenos Aires, Argentina.

Franco, N. V., Castro, A., Cardillo, M., \& Charlin, J. (2009). La importancia de las variables morfológicas, métricas y de microdesgaste para evaluar las diferencias en diseños de puntas de proyectil bifaciales pedunculadas: un ejemplo del sur de Patagonia continental. Magallania, 37(1), 99-112. doi: 10.4067/ S0718-22442009000100008

Gómez Otero, J., Banegas, A., Goye, M. S., \& Franco, N. V. (2011, enero). Variabilidad morfológica de puntas de proyectil en la costa centro-septentrional de Patagonia Argentina: primeros estudios y primeras preguntas. In VIII Congreso de Historia Social y Política de la Patagonia Argentino - Chilena: las fuentes en la construcción de una historia patagónica. Rawson-Chubut.

Gradin, C. J. (1971). Parapetos habitacionales en la meseta Somuncurá Provincia de Río Negro. Relaciones de la Sociedad Argentina de Antropología, 5-2, 171-185. 
Gradin, C. J. (1980). Secuencias radiocarbónicas del sur de la Patagonia argentina. Relaciones de la Sociedad Argentina de Antropología, 14(1), 177-194.

Hermo, D. O., \& Terranova, E. D. (2016). Distribución de núcleos en el sector central de la mesta de Somuncurá (Río Negro, Argentina). Relaciones entre la base regional de recursos líticos y el aprovisionamiento de materias primas líticas. Arqueología, 22(2), 291-310.

Hocsman, S. (2007). Producción de bifaces y aprendices en el sitio Quebrada Seca 3 - Antofagasta de la Sierra, Catamarca (55004500 años ap.). In A. E. Nielsen, M. C. Rivolta, V. Seldes, M. M. Vázquez \& P. H. Mercolli (Comps.), Producción y circulación prehispánicas de bienes en el sur andino (pp. 55-82). Córdoba: Editorial Brujas.

Hocsman, S. (2010). Cambios en las puntas de proyectil durante la transición de cazadores-recolectores a sociedades agropastoriles en Antofagasta de la Sierra (Puna argentina). Arqueología, (16), 59-86.

Hocsman, S. (2011). La transición de la caza-recolección a la producción agropastoril en Antofagasta de la Sierra (Puna Meridional Argentina) vista desde la tecnología lítica. Chungara Revista de Antropología Chilena. En referato.

Hughes, S. S. (1998). Getting to the point: evolutionary change in prehistoric weaponry. Journal of Archaeological Method and Theory, 5(4), 345-408.

Ingold, T. (1993). The temporality of the landscape. World Archaeology, 25(2), 152-174.

Keeley, L. H. (1980). Experimental determination of stone tool uses: a microwear analysis. Chicago: University of Chicago Press.

Lynch, V., \& Terranova, E. (2017, noviembre). Lithic micro-wear analysis of Late Holocene tools from Laguna Azul locality (Somuncurá plateau, Rio Negro, Argentina). In 11th International Symposium on knappable materials: from tool-stone to stone tools, Instituto Multidisciplinario de Historia y Ciencias Humanas, Buenos Aires.

Lynch, V., \& Terranova, E. (2019). A traceological approach to the use of Laguna Azul during the Late Holocene (from ca. 2000 years BP) in Norpatagonia, Argentina. Archaeoloical and Anthropological Sciences, 11(8), 4157-4169. doi: 10.1007/ s12520-019-00806-7

Lynch, V., Vargas Gariglio, J., \& Terranova Enrique, D. (2019). Engraved stone plaquettes from the North Patagonian area (Somuncurá plateau, Río Negro, Argentina) and the use of different microscopic techniques for their analysis. World Archaeology, 51(1), 104-125. doi: 10.1080/00438243.2018.1542340

Mansur-Franchomme, M. E. (1983). Traces d'utilisation et technologie lithique: exemple de la Patagonie (Tesis de doctorado). Universidade Bordeaux, França.
Martínez, J. G. (2003). Ocupaciones humanas tempranas y tecnología de caza en la microrregión de Antofagasta de la Sierra (10.000 a 7.000 AP) (Tesis de doctorado). Instituto Miguel Lillo, Universidad Nacional de Tucumán, Tucumán, Argentina.

Miotti, L., Blanco, R. V., Terranova, E., Marchionni, L., Hermo, D., \& Mosquera, B. (2014). La naturaleza de la observación: evidencias arqueológicas en Somuncurá (Río Negro). In G. Cassiodoro, A. Re \& D. Rindel (Eds.), Integración de diferentes líneas de evidencia en la Arqueología Argentina (pp. 73- 91). Buenos Aires: Aspha.

Miotti, L., Terranova, E., Blanco, R. V., Marchionni, L., Hermo, D., \& Magnin, L. (2016). Entre basaltos y lagunas: las estructuras de piedra de la meseta de Somuncurá. Apuntes para la reflexión de los patrones de movilidad de los cazadores-recolectores. In Arqueología de la Patagonia: de Mar a Mar (pp. 256-266). Santiago de Chile: CIEP.

Moreno, F. P. (n.d.). [s.f.]. Recuerdos de un viaje a Nahuel Huapi: 1) El llano de Yamnagóo. Visita a los Guenaken. Una raza que muere. Buenos Aires: Biblioteca del Museo Etnográfico.

Nami, H. G. (1993-1994). Aportes para el conocimiento de técnicas líticas del Pleistoceno final. Análisis de artefactos bifaciales del norte de Venezuela (colección Edmonton, Canada). Relaciones de la Sociedad Argentina de Antropología, 19, 417-449.

Odell, G. H., \& Cowan, F. (1986). Experiments with spears and arrows on animal targets. Journal of Field Archaeology, 13(2), 195-212. doi: 10.2307/530220

Prates, L. (2010). La ocupación humana prehispánica del valle del río Negro. In R. Masera (Coord.), Los ríos mesetarios norpatagónicos: aguas generosas del Ande al Atlántico (pp. 339359). Río Negro: Edición del Gobierno de Río Negro.

Ratto, N. (1994). Funcionalidad versus adscripción cultural: cabezales líticos de la margen norte del estrecho de Magallanes. In J. Lanata \& L. Borrero (Comps.), Arqueología de Cazadores-recolectores: límites, casos y aperturas (Arqueología Contemporánea, 5) (pp. 105-120). Buenos Aires: Programa de Estudios Prehistóricos.

Ratto, N. (2003). Estrategias de caza y propiedades del registro arqueológico en la Puna de Chaschuil (Tesis de doctorado). Facultad de Filosofía y Letras, Universidad de Buenos Aires, Depto Tinogasta, Catamarca, Argentina.

Ratto, N. (2013). Diversidad de tecnologías de caza en la puna transicional de Chaschuil (Dpto. Tinogasta, Catamarca). Comechingonia. Revista de Arqueología, 17(1), 85-103.

Remesal, M., Salani, F., Franchi, M., \& Ardolino, A. (2001). Hoja Geológica 4169-IV, Maquinchao. Provincia de Río Negro (Boletín, No 32). Buenos Aires: Instituto de Geología y Recursos Minerales, Servicio Geológico Minero Argentino. 
Rosero Moreno, E. A. (2011). Tecnología de caza en Antofalla, Departamento de Antofagasta de la Sierra, Catamarca. Revista del Museo de Antropología, 4(1), 17-32.

Shott, M. J. (1997). Stones and shafts redux: the metric discrimination of chipped-stone dart and arrow points. American Antiquity, 62(1), 86-101. doi: 10.2307/282380

Sidéra, I., Vila, E., \& Erikson, P. (2006). La chasse: pratiques sociales et symboliques (Collection Colloques de la Maison RenéGinouvès, No. 2). Paris: De Boccard.

Stern, C. R., Gómez Otero, J., \& Belardi, J. B. (2000). Características químicas, fuentes potenciales y distribución de diferentes tipos de obsidianas en el norte de la provincia del Chubut, Patagonia Argentina. Anales del Instituto de la Patagonia, 28, 275-290.
Terranova, E. (2013). Arqueología de la cuenca del arroyo Talagapa, meseta de Somuncurá (Provincia de Río Negro) (Tesis de doctorado). Facultad de Ciencias Naturales y Museo, Universidad Nacional de La Plata, La Plata, Buenos Aires, Argentina.

Vargas Gariglio, J., Terranova, E., \& Lynch, V. (2019). Estudio preliminar del parapeto 3 de Laguna Azul, Meseta de Somuncurá (Río Negro, Argentina). In J. Gómez Otero, A. Svoboda \& A. Benegas (Eds.), Arqueología de Patagonia: El pasado entre las arenas (pp. 443-454). Puerto Madryn: Instituto de Diversidad y Evolución Austral.

\section{CONTRIBUIÇÃO DOS AUTORES}

Virginia Lynch contribuiu com conceituação, análise formal, investigação, metodologia e escrita - rascunho original; Darío Omar Hermo com conceituação e escrita - rascunho original; e Laura Lucía Miotti com conceituação e escrita - rascunho original. 
